\title{
Statistical Characterization and Capacity of Shallow Water Acoustic Channels
}

\author{
Andreja Radosevic \\ University of California, San Diego \\ 9500 Gilman Drive \\ La Jolla, CA 92093-0407 \\ Email: aradosevic@ucsd.edu
}

\author{
John G. Proakis \\ University of California, San Diego \\ 9500 Gilman Drive \\ La Jolla, CA 92093-0407 \\ Email: jproakis@ucsd.edu
}

\author{
Milica Stojanovic \\ Northeastern University \\ 360 Huntington Avenue \\ Boston, MA 02115 \\ Email: millitsa@ece.neu.edu
}

\begin{abstract}
This paper focuses on statistical characterization and capacity evaluation of shallow water acoustic communications channels. Wideband single-carrier and multi-carrier probe signals are employed to measure the time-varying channel response, and to estimate its statistical properties that play an important role in the design of bandwidth-efficient and powerefficient communication systems. To assess the capacity of an underwater acoustic channel, we first assess the probability density functions that model the signal fading. Rician fading is found to provide a good match for the experimental data collected in the Pacific Ocean during the summer 2008 Kauai acoustic communications experiment (KAM'08). This model is used to evaluate the ergodic channel capacity and the related bandwidth efficiency for both single-input single-output (SISO) and multiple-input multiple-output (MIMO) systems.
\end{abstract}

\section{INTRODUCTION}

An underwater acoustic (UWA) channel is an extremely complex communication environment characterized by frequency-dependent path loss, multipath propagation and low speed of sound (approx. $1500 \mathrm{~m} / \mathrm{s}$ ). Moreover, the channel impulse response is sparse and each channel path acts as a time-varying low-pass filter with additional Doppler shifting and spreading [1]. Due to the large delay spreads $(10-100 \mathrm{~ms})$ an UWA channel exhibits frequency selectivity which affects the wideband acoustic communications signals. Because of the extreme channel complexity, design and simulation of UWA communication systems requires accurate channel modeling. Ray theory can be used to provide a deterministic description of the (average) multipath propagation, but it must be augmented by a statistical description of the random channel variations in time. A complete model must take into account the frequency-dependent path loss, channel sparseness, and Doppler shifting and spreading effects.

Proper design of channel probe signals is a prerequisite for reliable statistical characterization of an UWA channel. We designed channel probes in two forms: single-carrier (SC) and multi-carrier (MC) probes. By examining the channel impulse and frequency response, as well as correlation functions in time, important channel parameters were extracted; namely, the channel coherence time and coherence bandwidth [2].

This work is supported by the Multidisciplinary University Research Initiative (MURI) ONR Grants N00014-07-1-0739 and N00014-07-1-0738.
Candidate statistical models that were considered for the signal fading include Rician, Nakagami and log-normal distributions. Due to the non-stationarity of the channel, analysis is constrained to short-term statistical characterization. Hence, the statistical properties of the channel fading, as well as the parameters of the underlying statistical model are subject to the evolution in time (i.e., for the Rician fading scenario, the Rician $k$-factor, the mean and the standard deviation of the underlying complex normal distribution can change in time). This is an important observation since the assessment of the channel capacity, especially for MIMO systems, depends on how well the fading statistics can be estimated. Moreover, because of the non-stationarity, design of the capacity-achieving adaptive coding/modulation is of particular interest, and it is based on the ability of the communication system to track the channel model parameters.

There are few available analyses regarding the capacity of the UWA channel. Earlier work, given in [3] and [4], considered a time-invariant channel model with additive Gaussian noise (possibly not white) and a Rayleigh fading model with additive white Gaussian noise (AWGN), respectively. In [5], a ray theory based model is proposed where the individual multipath arrivals are modeled as Rayleigh random processes with non-Gaussian ambient noise. Neither of these analyses relates the distance to the capacity of the UWA channel. In a recent work [6], an analytical method is proposed which takes into account the physical laws of acoustic propagation and ambient noise. We use the results of this work to obtain semianalytical solutions for the average capacity of the recorded time-varying channels.

Since the analysis of MIMO capacity of the UWA channel remains an open area, information rate limitations are discussed based on recent results in information theory. It is shown in [7], [8] that significant capacity improvements can be obtained by using MIMO systems and space-time coding. Moreover, for a flat Rayleigh fading channel, it is known that if the number of transmitter and the receiver elements is the same, the ergodic capacity increases linearly with this number. This result can be extended for any fading distribution under additive white Gaussian noise (AWGN). The amount of increase in the information rate, i.e. the spatial multiplexing gain, depends primarily on the channel fading 
distribution. This is an important observation for systems that do not exhibit Rayleigh fading, such as mobile-terrestrial radio systems where a line-of-sight component results in Rician fading, or, as we shall see, acoustic channels. Ergodic capacity results can also be extended to frequency-selective fading channels [9]-[11].

In this paper we consider statistical modeling of UWA channels with the goal of assessing their ergodic capacity. Our focus is on a set of measured channel responses, obtained from the signals recorded during the Kauai acoustic communications (KAM'08) experiment. We begin in Section II by describing the channel probe signals used in the experiment, and the resulting measurements of the time-varying channel responses. In Section III, we discuss several statistical models for the channel fading: Rician, Nakagami and log-normal. Also, we examine the correlation properties of the channel in time and assess the channel spectral efficiency. Channel capacity is addressed in Section IV, both for the MISO and the MIMO setting. The capacity analysis of flat and frequency-selective fading systems is based on distinguishing two scenarios with different amount of channel state information (CSI) at the transmitter: (1) no CSI, and (2) partial CSI, where only the statistical properties of the channel, but not the actual channel realizations, are known to the transmitter. Due to the long propagation delay, only the second scenario is deemed realistic in a general UWA channel. Concluding remarks are provided in Section V.

\section{Channel Measurements}

A shallow water communication channel is characterized by rapid phase variations, severe frequency-selectivity and long delay spread. These facts influence the design of channel probe signals. Two types of signals were designed for the present experiment: single-carrier (SC) and multi-carrier (MC) probes.

Single-Carrier probes were designed for the total bandwidth of $B=7812 \mathrm{~Hz}$ centered around $f_{c}=16 \mathrm{kHz}$. To allow for computationally efficient coherent detection, SC probes are kept short. A 28-element Turyn sequence [13], which has good auto-correlation properties, was chosen for this reason. Square root raised cosine pulse shaping is applied with the roll-off factor $\alpha=0.25$. At the chip rate of $6250 \mathrm{bps}$, the duration of one sequence is $4.48 \mathrm{~ms}$. Pseudo-noise properties of the modulated sequence lead to an almost flat spectrum of the probe signal over the signal bandwidth, thus allowing reliable channel estimation. The channel probe signal was transmitted during a 57 s period of time, with 366 repeated transmissions, each transmission separated by the silence periods of $150 \mathrm{~ms}$. The digitally generated probe signal is sampled at $f_{s}=100$ $\mathrm{kHz}$. Coherent detection at the receiver is performed via matched filtering to the transmitted probe signal, giving an estimate of the channel impulse response.

Multi-carrier modulation was considered as an alternative to single-carrier wideband modulation for channels with frequency-selective distortion. By using rectangular pulse shaping in time, multi-carrier modulation and detection are efficiently implemented using the Fast Fourier Transform in the
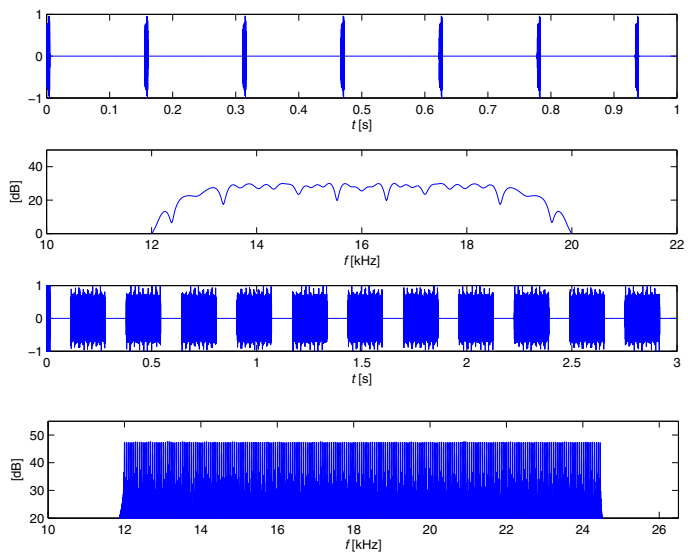

Fig. 1. Channel probe signals: single-carrier probes in time and their spectrum are shown in the top two graphs; multi-carrier probes are shown below.

form of orthogonal frequency division multiplexing (OFDM). Our MC channel probe is based on modulating every 8-th sub-carrier of the OFDM signal by a pseudo-noise sequence, and leaving the remaining sub-carriers empty. In this manner, the inter-carrier interference is sufficiently reduced, so that it will not present a problem for frequency-domain channel estimation. The OFDM signal bandwidth is $B=12.5 \mathrm{kHz}$ (frequency of the first sub-carrier is $f_{0}=12 \mathrm{kHz}$ ), the total number of sub-carriers is $K=2048$ with frequency spacing of $\Delta f=6 \mathrm{~Hz}$, and the signal duration is $T=160 \mathrm{~ms}$. Similarly as in the SC case, the overall probe duration is $57 \mathrm{~s}$ with 216 transmission blocks, and each transmission is followed by a silence interval of $100 \mathrm{~ms}$. Again, the sampling frequency of the digitally generated probe signal is $f_{s}=100 \mathrm{kHz}$. Figure 1 shows the spectral characteristics of the SC and MC probes. Figure 2 shows the estimates of the channel impulse response (magnitude) obtained from the data collected during KAM'08 experiment. The experiment was conducted off the western side of Kauai Island, Hawaii, in the Pacific Ocean, in $100 \mathrm{~m}$ deep water and $4 \mathrm{~km}$ transmission distance. The transmitter and the receiver were deployed as 8-element (7.5 m spacing) and 16 -element ( $3.75 \mathrm{~m}$ spacing) arrays, respectively. Results are illustrated for transmission between the fourth transducer from the bottom ( $49.5 \mathrm{~m}$ depth) and the fourth hydrophone from the bottom ( $87.25 \mathrm{~m}$ depth). It is assumed that both the source and the receiver are fixed, and no significant Doppler spreading and shifting were observed. The different peaks in the channel estimates can be associated with multiple surface and bottom reflections calculated from the geometry of the experiment. Note how these significant paths vary in time due to the changing conditions in the channel over the one minute observation interval. This fact necessitates short-term statistical characterization of the channel fading, since the energy content of the significant paths can dramatically change during longer observation intervals. The next section will focus on this problem. From Fig.2 we note that the channel transfer 

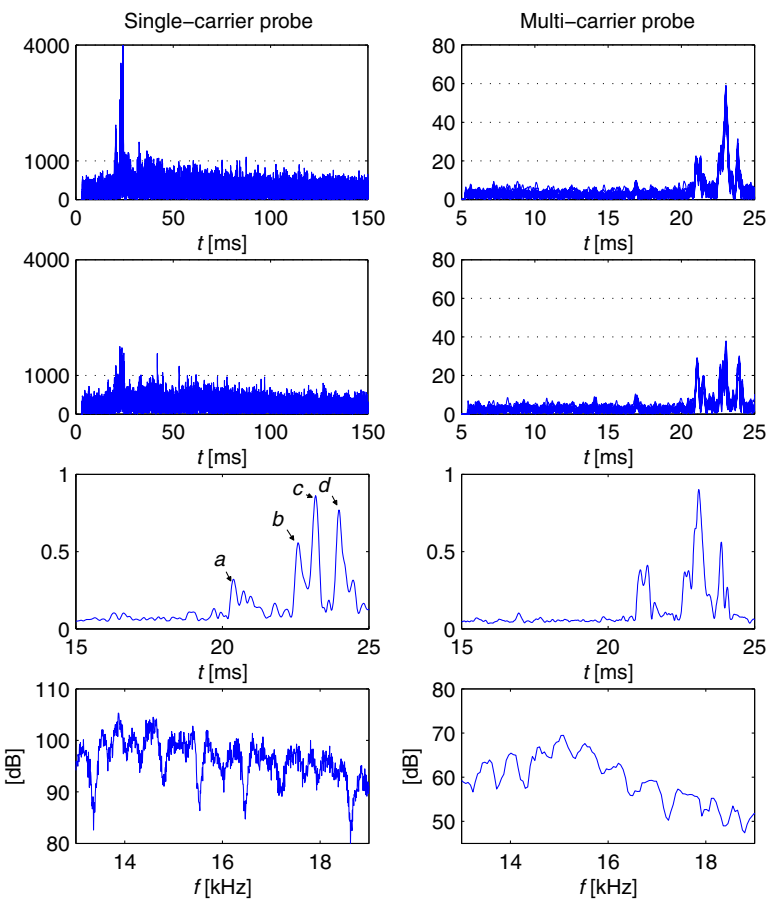

Fig. 2. Channel estimates: shown in the top two rows are the ensembles of 12 consecutive channel responses in the time; shown below are the top row ensemble averages in the time and frequency domain.

functions obtained from the MC probes are smooth compared to those obtained from the SC probes. This is an artifact of insufficient resolution in the frequency domain of the probe signal employed. The lack of precision in the estimated frequency response is clearly seen through more spectral flatness in comparison to the estimate based on wideband SC probing. This is reflected in the time domain as the lower resolution in the impulse response, which prevents us from distinguishing adjacent propagation paths. Thus, the MC signal may not be able to capture the frequency-selectivity of the channel, and cannot be used to assess its coherence bandwidth. With 256 active sub-carriers, our MC probes are limited to a frequency resolution of $48 \mathrm{~Hz}$, which corresponds to about $20 \mathrm{~ms}$ of multipath spread. Hence, any delay spreading longer than 20 ms will not be accurately captured. In fact, we note from the SC probes that the multipath spread $T_{m}$ is on the order of 100 $\mathrm{ms}$ (coherence bandwidth of $10 \mathrm{~Hz}$ ). Large multipath spread is the result of appearance of the occasional and unstable arrivals in the estimates of the individual channel realizations for the observation interval between 80 and $120 \mathrm{~ms}$. Increasing the frequency resolution of the $\mathrm{MC}$ probes would require an increase in the number of sub-carriers; however, the resulting increase in the signal duration would challenge the detection capability on a time-varying channel. Nonetheless, the note that MC probes may be useful for assessing the time-coherence properties of the channel in each narrow sub-band.

Based on the above discussion, we will use the SC channel measurement in what follows, due to its advantages in both time and frequency domains, namely, short signal duration (well below the coherence time of the channel) allows simple coherent detection, while signal flatness in the frequency domain gives accurate estimates of the channel coherence bandwidth and ensures sufficient resolution in the impulse response.

\section{Statistical Characterization}

Our statistical characterization focuses on two aspects: the probability density function (pdf) of the path responses, and their time-correlation properties. Because the channel is not stationary, we focus on short-term characterization (order of seconds). By assuming a quasi-stationary channel, and looking at its short-term characteristics, we will later assess the channel capacity. Note that the selection of the window of (assumed) stationarity is very important, as it has a profound impact on the analysis to follow.

\section{A. Channel fading distribution}

An underwater channel is characterized by frequencydependent path loss and rapid random fluctuations that cause variation in the path loss. Moreover, dynamical changes of the environmental profile are severe (i.e., random motion of the surface reflection points) suggesting several possible models for the fading distribution.

Figure 3 illustrates the time variation of the significant paths labeled as $a, b, c$ and $d$ in Figure 2. A careful examination of this figure underscores the need for short-term characterization of the channel. For example, note that the channel tap $a$ behave differently in the time intervals from 0 to $30 \mathrm{sec}$ and from 30 to $50 \mathrm{sec}$. The change in the local mean indicates a non-stationary random process. On the other hand, note that quasi-stationary intervals for the path $b$ are on the order of 10 seconds for the time interval from 0 to $20 \mathrm{sec}$ and on the order of seconds for the time interval from 20 to $50 \mathrm{sec}$. To account for this fact, we have chosen a window of 2 seconds to represent the quasi-stationary period for the models that we will consider. Since the behavior of different paths can be characterized using longer intervals of quasi-stationarity than the adopted window size, for the purpose of histogram presentation, we can superimpose several consecutive windows and analyze their statistical properties jointly (i.e., for tap $a$ we can observe jointly up to 10 windows, and for tap $b$ up to 10 windows for the time interval 0 to $20 \mathrm{sec}$ ). Figure 4 shows how the Rician, Nakagami and log-normal pdfs fit when superimposed on top of the measurement histograms. The pdfs of the three distributions are given by the following expressions:

$$
\begin{gathered}
f_{X_{\text {rice }}}\left(x_{r}\right) \sim 2 \frac{(1+k)}{\Omega} x_{r} e^{-\frac{(1+k)}{\Omega} x_{r}^{2}-k} I_{0}\left(2 \sqrt{\frac{k(1+k)}{\Omega}} x_{r}\right), \\
f_{X_{\text {naka }}}\left(x_{n}\right) \sim 2\left(\frac{m}{\omega}\right)^{m} \frac{x_{n}^{2 m-1}}{\Gamma(m)} e^{-\frac{m x_{n}^{2}}{\omega}}, x_{n} \geq 0, \\
f_{X_{\text {logn }}}\left(x_{l}\right) \sim \frac{1}{x_{l} \sigma \sqrt{2 \pi}} e^{-\frac{\left(\log \left(x_{l}\right)-\mu\right)^{2}}{2 \sigma^{2}}}, x_{l}>0,
\end{gathered}
$$



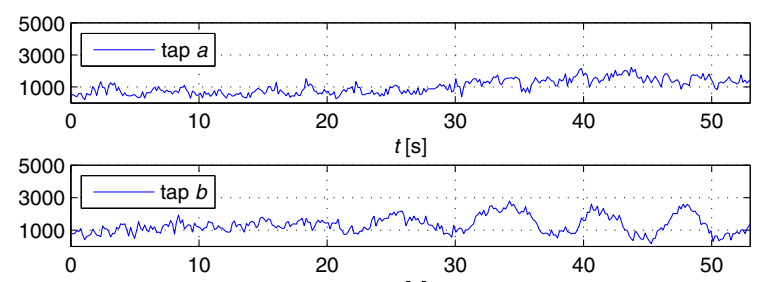

$t[\mathrm{~s}]$

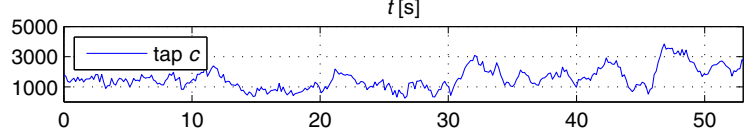

$t[\mathrm{~s}]$

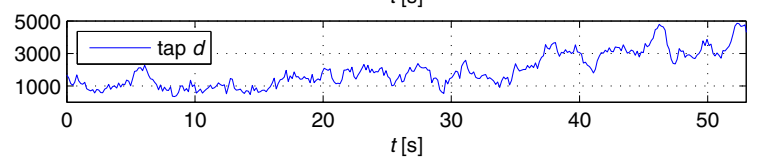

$t[\mathrm{~s}]$

Fig. 3. Magnitudes of the channel propagation paths $(a, b, c$ and $d$ from Fig.2) vs. time.

where $I_{0}$ in Eq.(1) is the zeroth-order modified Bessel function of the first kind, $E\left\{X_{\text {rice }}^{2}\right\}=\Omega$, and $k$ is the Rician factor. In Eq.(2), $m$ is the shape parameter, and $\omega$ is the spreading factor. In Eq. (3), $\sigma$ and $\mu$ are the standard deviation and the mean value of the underlying normal distribution of $\log \left(X_{\log n}\right)$.

The parameters of the Nakagami and log-normal pdf have been obtained based on the estimated parameters of the fitted Rician distribution (from the data histograms in Fig. 4). These parameters have been determined to yield equivalent second and fourth moment of the underlying random variables:

$$
\begin{gathered}
E\left\{X_{\text {rice }}^{2}\right\}=E\left\{X_{\text {naka }}^{2}\right\}=E\left\{X_{\text {logn }}^{2}\right\}=1, \\
\frac{\left(E\left\{X_{\text {rice }}^{2}\right\}\right)^{2}}{E\left\{X_{\text {rice }}^{4}\right\}-\left(E\left\{X_{\text {rice }}^{2}\right)^{2}\right.}= \\
=\frac{\left(E\left\{X_{\text {naka }}^{2}\right\}\right)^{2}}{E\left\{X_{\text {naka }}^{4}\right\}-\left(E\left\{X_{\text {naka }}^{2}\right\}\right)^{2}}= \\
=\frac{\left(E\left\{X_{\text {logn }}^{2}\right\}\right)^{2}}{E\left\{X_{\text {logn }}^{4}\right\}-\left(E\left\{X_{\text {logn }}^{2}\right\}\right)^{2}} .
\end{gathered}
$$

This normalization is performed in order to keep the mean value of the log-normal random variable in the range of mean values of the Rician and Nakagami fading, since the conditions $E\left\{X_{\text {rice }}\right\}=E\left\{X_{\text {naka }}\right\}=E\left\{X_{\text {logn }}\right\}$ and $E\left\{X_{\text {rice }}^{2}\right\}=$ $E\left\{X_{\text {naka }}^{2}\right\}=E\left\{X_{\text {logn }}^{2}\right\}$ cannot be satisfied simultaneously. It remains unanswered which physical process could be a possible cause of these two fading pdfs.

\section{B. Equivalent Channel Model}

We define a time-varying discrete-time equivalent baseband channel model as

$$
h[n, m]=\sum_{l} h_{l}[m] \delta[n-l],
$$

where $h[n, m]$ is the response of the channel at time $m$ due to an impulse applied at time $m-n$. The channel tap gains, $\left\{h_{l}\right\}$,
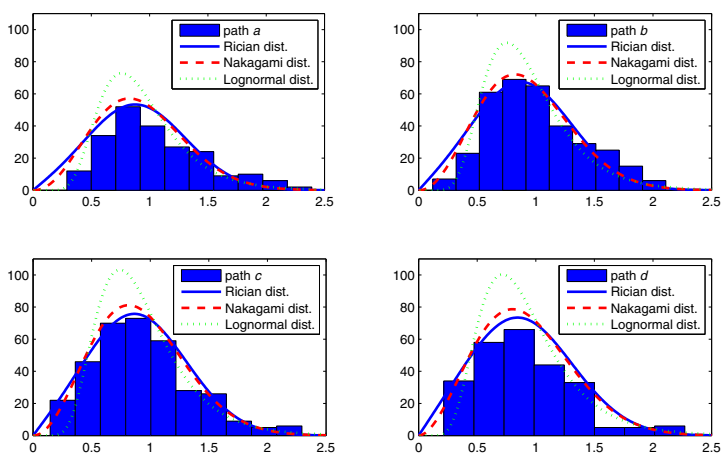

Fig. 4. Histogram and fit of the magnitude of the channel propagation path. Each histogram is based on observations taken over 10 seconds.

do not necessarily represent the actual physical propagation paths. Each tap gain $h_{l}$ can contain in itself the contributions of all the physical paths; the exact relationship depends on the system bandwidth [1]. Since the channel impulse response is sparse, these contributions are small in a wideband system, and the dominant coefficients of the uniformly spaced equivalent model (6) can be roughly associated with the physical propagation paths.

We initially assume the Rician fading model where $h_{l}$ 's are complex Gaussian random variables with independent real and imaginary parts, distributed as $\mathcal{N}\left(\mu_{l} / \sqrt{2}, \sigma_{l}^{2}\right)$. The equivalent Nakagami and log-normal fading models can be derived from expressions (4) and (5). Assuming normalization to the lognormal fading, $E\left\{\left|h_{l}\right|^{2}\right\}=\Omega_{l}=\mu_{l}^{2}+2 \sigma_{l}^{2}$ and $\sum_{l} \Omega_{l}=1$, we have that each channel tap is given as

$$
h_{l}[m]=\sqrt{\frac{\Omega_{l} k_{l}}{k_{l}+1}} \frac{(1+i)}{\sqrt{2}}+\sqrt{\frac{\Omega_{l}}{k_{l}+1}} \frac{1}{\sqrt{2}} h_{l 0}[m],
$$

where $h_{l 0}$ is a complex Gaussian random variable with independent real and imaginary parts, each distributed as $\mathcal{N}(0,1)$, and the Rician $k$-factor is defined as the ratio of the power in the mean value to the power in the diffuse component:

$$
k_{l}=\frac{\mu_{l}^{2}}{2 \sigma_{l}^{2}} \text {. }
$$

\section{Measurement of the channel coherence time}

Experimental measurements of the correlation in time of the channel impulse response give us an estimate of the channel coherence time. For a reliable estimate, the channel probe signal must be significantly shorter in duration than the channel coherence time. We also note here that Doppler shifting due to the transmitter and the receiver motion should be distinguished from Doppler spreading caused by channel motion and variation.

The normalized correlation function is defined as

$$
\rho[n]=\frac{1}{\left|A_{m, m+n}\right|} \sum_{A_{m, m+n}} \frac{\left|\sum_{k} h^{*}\left[k, t_{m}\right] h\left[k, t_{m+n}\right]\right|}{\sqrt{\sum_{k}\left|h\left[k, t_{m}\right]\right|^{2} \sum_{k}\left|h\left[k, t_{m+n}\right]\right|^{2}}},
$$




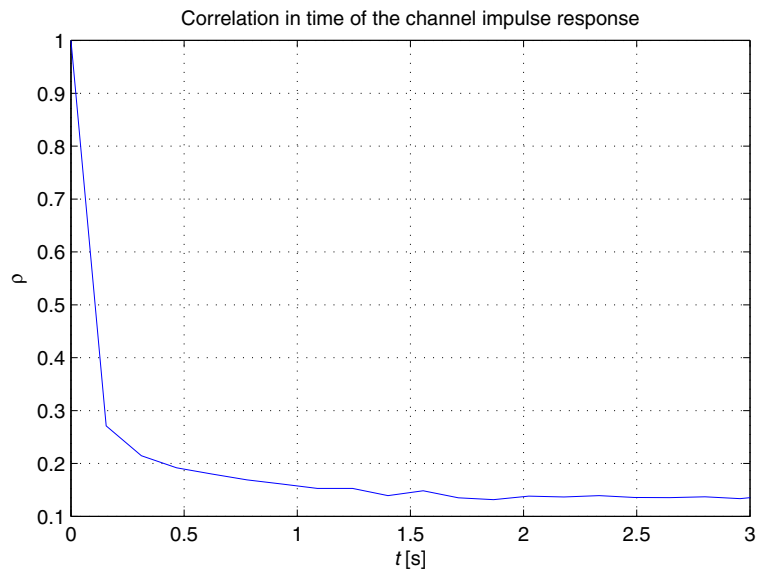

Fig. 5. Correlation factor.

where $A_{m, m+n}$ is the set of all independent pairs of indices $(m, m+n)$ such that if $n=1$ then $A_{1}=$ $\{(m, m+1):(1,2),(3,4), \ldots\} \cdot t_{m+n}=(m+n) \Delta L$ where $\Delta L$ is the discrete-time repetition interval of the transmitted probe sequence (e.g., for the single-carrier probe signal $\Delta L=$ 15561 samples at $f_{s}=100 \mathrm{kHz}$ ). Note that the correlation coefficient will also depend on the time index $m$ as the channel is non-stationary, but we omit this dependence with the understanding that the results will be illustrated only for a quasi-stationary interval on the order of seconds. In Figure 5 we see an experimental measurement of this channel parameter. From this figure, the coherence time is estimated to be $T_{c} \approx 200 \mathrm{~ms}$. Since our probe signals are of duration $T=4.5 \mathrm{~ms}$, the assumption that $T<<T_{c}$ is justified.

\section{Spectral efficiency}

We assess the spectral efficiency of the channel according to the method proposed in [6]. This method makes use of an approximate model which gives the ambient noise power spectral density as

$$
10 \log N(f)=n_{0}-18 \log f[\mathrm{~dB}],
$$

where the level $n_{0}$ is subject to adjustment for a particular UWA channel of interest. The Welch power spectral density estimate [2] of the ambient noise, obtained from the experimental recordings during the silence periods, is shown in Fig.6 along with the model (10). This figure also illustrates the histogram of the recorded ambient noise, indicating that this noise can be modeled as Gaussian. Given this noise model, the short-term channel capacity can be computed as

$$
C\left(t_{k}^{*}\right)=\sum_{i} \Delta f \log _{2}\left(1+\frac{S\left(f_{i}\right)\left|H\left(f_{i}, t_{k}^{*}\right)\right|^{2}}{N\left(f_{i}\right)}\right),
$$

where $t_{k}^{*}$ denotes the $k$-th measurement interval of 12 consecutive transmissions of the probe signal (i.e., $t_{k}^{*}$ is discrete-time interval from $12(k-1) \Delta L$ to $12 k \Delta L-1) . \Delta f$ is a narrow band of frequencies centered at frequency $f_{i}$, the index $i$ ranges across the given signal bandwidth, and $\left.\mid H\left(f, t_{k}^{*}\right)\right) \mid$ is the shortterm estimate of the channel transfer function magnitude for
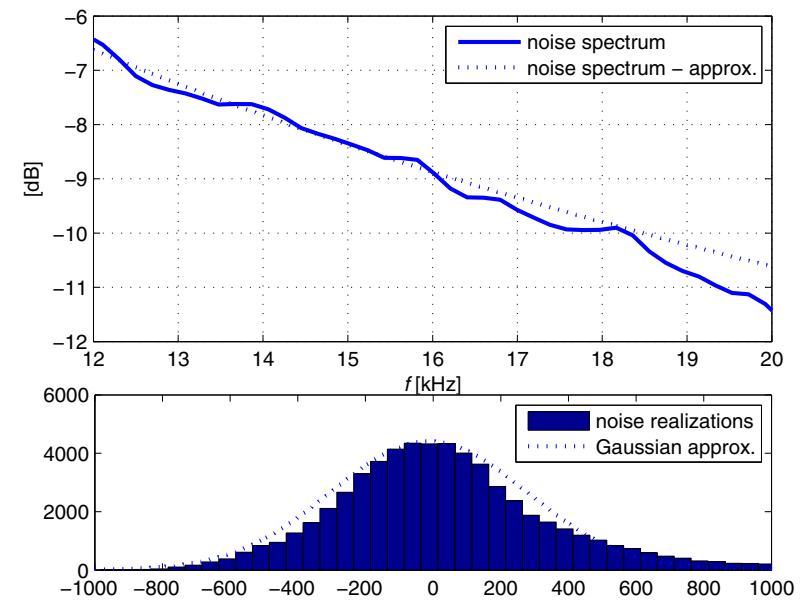

Fig. 6. Ambient noise spectrum and histogram.

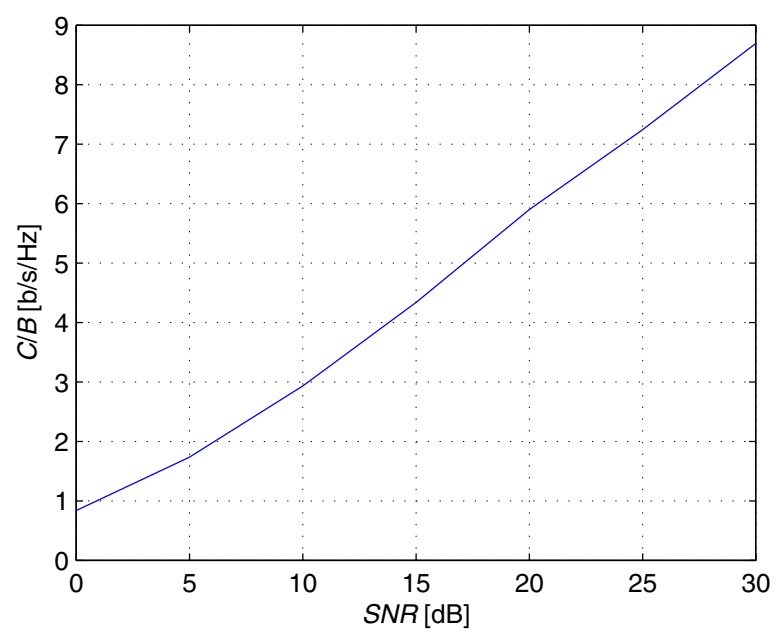

Fig. 7. Average spectral efficiency.

the time interval $t_{k}^{*} . S(f)$ is the spectrum of the transmitted signal which maximizes the channel capacity subject to the constraint on finite transmission power. The optimal spectrum allocation is determined by the water-filling algorithm [6].

Averaging over all short-term estimates of the channel capacity, we obtain an estimate of the ergodic capacity

$$
C=\frac{1}{K} \sum_{k=1}^{K} C\left(t_{k}^{*}\right),
$$

where $K$ is the number of measurement intervals. The spectral efficiency is now obtained by normalizing the ergodic capacity by the bandwidth. Figure 7 illustrates the result of averaging the spectral efficiency over $K=30$ realizations of $\left.\mid H\left(f, t_{k}^{*}\right)\right) \mid$ obtained from measurements of the channel impulse responses in a $57 \mathrm{~s}$ time interval. 


\section{ERGODIC CAPACITY}

For the MIMO system configuration, we assume a spatially correlated channel with $N_{t}$ transmit and $N_{r}$ receive elements. The channel matrix of size $N_{t} \times N_{r}$ is given by

$$
\mathbf{H}[n, m]=\sum_{l} \mathbf{H}_{l}[m] \delta[n-l]
$$

where $\mathbf{H}_{l}$ is the $l$-th tap gain matrix, containing $N_{t} \times N_{r}$ identically distributed elements which are defined similarly as the $1 \times 1$ coefficients $h_{l}$ in the expression (7). Therefore, we have that

$$
\mathbf{H}_{l}[m]=\sqrt{\frac{\Omega_{l} k_{l}}{k_{l}+1}} \overline{\mathbf{H}}_{l}+\sqrt{\frac{\Omega_{l}}{k_{l}+1}} \frac{1}{\sqrt{2}} \widetilde{\mathbf{H}}_{l}[m],
$$

where $\Omega_{l}$ represents the multipath delay profile of the channel, $\overline{\mathbf{H}}_{l}$ is a rank-1 matrix of mean values with all elements equal to $(1+i) / \sqrt{2}$, and $\widetilde{\mathbf{H}}_{l}=\boldsymbol{\Theta}_{R, l}^{1 / 2} \widetilde{\mathbf{H}}_{\omega, l} \boldsymbol{\Theta}_{T, l}^{1 / 2}$. Here, $\widetilde{\mathbf{H}}_{\omega, l}$ is $N_{t} \times$ $N_{r}$ matrix with independent and identically distributed (i.i.d.) complex Gaussian random variables with independent real and imaginary parts, each distributed as $\mathcal{N}(0,1)$, and $\boldsymbol{\Theta}_{R, l}$ and $\boldsymbol{\Theta}_{T, l}$ are the receive and the transmit correlation matrices of the $l$-th tap. We assume that different taps are uncorrelated, i.e. the only correlation in the channel is among elements of the $l$-th tap, which is defined by $\boldsymbol{\Theta}_{R, l}$ and $\boldsymbol{\Theta}_{T, l}$. Note that in the expression (13) we have a uniform tap spacing as in the SISO case.

We will discuss several cases: flat-fading and frequencyselective fading, various fading distributions, and various levels of the transmitter CSI. Since the complete CSI knowledge at the transmitter is not a realistic scenario, our focus will be on the following two cases: no knowledge, and only statistical knowledge of the channel at the transmitter side.

\section{A. Flat-fading}

From the expression (13), a flat-fading channel model is obtained when only a single tap is taken. Omitting the tap's index, the system model is given by

$$
\mathbf{y}[n]=\mathbf{H}[n] \mathbf{x}[n]+\mathbf{w}[n],
$$

where $\mathbf{x}[n]$ is an $N_{t} \times 1$ is input vector, $\mathbf{y}[n]$ is an $N_{r} \times 1$ receive vector, and $\mathbf{w}[n]$ is an $N_{r} \times 1$ vector of the circularly symmetric complex Gaussian noise. The ergodic capacity of the channel can be computed as the maximum of the mutual information,

$$
C_{N_{t}, N_{r}}=\max _{p(\mathbf{x}), E\left\{\mathbf{x}^{H} \mathbf{x}\right\} \leq P} I(\mathbf{x} ; \mathbf{y}),
$$

where $p(\mathbf{x})$ is the pdf of the input signal vector $\mathbf{x}$ that satisfies a power constraint $E\left\{\mathbf{x}^{H} \mathbf{x}\right\} \leq P$. For the Gaussian noise channel, regardless of the channel fading distribution, the capacity-achieving distribution for the input signal, under the average power constraint, is circularly symmetric, zeromean complex Gaussian, as long as there is complete CSI at the receiver and statistical CSI at the transmitter [7], [8].
The covariance matrix $\mathbf{R}_{x x}=E\left\{\mathbf{x x}^{H}\right\}$ of the capacityachieving input signal depends on the fading distribution, which determines the flat-fading MIMO channel capacity as

$$
C_{N_{t}, N_{r}}=\max _{t r \mathbf{R}_{x x} \leq P} E_{\mathbf{H}}\left\{\log \operatorname{det}\left(\mathbf{I}_{N_{r}}+\mathbf{H} \mathbf{R}_{x x} \mathbf{H}^{H}\right)\right\},
$$

where $\mathbf{I}_{N_{r}}$ is the identity matrix of size $N_{r} \times N_{r}$ and $\operatorname{tr}(\cdot)$ denotes the trace of a matrix. If there is no CSI at the transmitter side, the capacity-achieving solution is $\operatorname{tr} \mathbf{R}_{x x}=$ $\sum_{i=1}^{N_{t}}\left|\left[\mathbf{R}_{x x}\right]_{i, i}\right|$, which is also the optimal solution for the Rayleigh fading case with statistical CSI at the transmitter [7]. In this case, equal power is allocated to all transmitters due to the lack of channel knowledge. For the case of Rician fading with limited (statistical) CSI, the capacity-achieving signal covariance remains unknown. In Ref. [12], the authors proposed a sub-optimal heuristic solution in the form

$$
\mathbf{R}_{x x}=\frac{P}{N_{t}(1+k)}\left(\mathbf{I}_{N_{t}}+k \mathbf{\Psi}_{N_{t}}\right),
$$

where $\boldsymbol{\Psi}_{N_{t}}$ is $N_{t} \times N_{t}$ is an $N_{t} \times N_{t}$ all-ones matrix. The idea behind this proposition is to exploit the knowledge of the Rician $k$-factor. If $k$ is zero, we have a Rayleigh fading channel and the optimal solution for the covariance matrix is an identity matrix, while if $k$ tends to infinity, we have a deterministic channel with AWGN, for which the optimal solution obtained by water-filling is the normalized matrix of all ones.

This suboptimal scheme is primarily designed to exploit the specular component in the Rican fading channel. For the Nakagami and log-normal models, the same scheme could cause severe performance degradation, and, hence, we do not emply it.

\section{B. Frequency-selective fading}

Frequency selective fading, which affects UWA channels, requires dealing with the channel memory. Our analysis is thus based on the observation of a blocks of $M$ input and receive signal vectors. To avoid inter-block interference among the received blocks, each input block is extended by a guard time (cyclic prefix) longer than the multipath spread. We also assume that $M>>T_{m} B$ and hence neglect the information rate loss incurred by insertion of the guard interval.

To assess the ergodic capacity of the Rician frequency selective channel, it is advantageous to represent a MIMO system by an input-output relationship

$$
\mathbb{Y}=\mathbb{H} \mathbb{X}+\mathbb{W}
$$

where $\mathbb{X}$ is an $M N_{t} \times 1$ space-time block vector of the input block stream, $\mathbb{Y}$ and $\mathbb{W}$ are $M N_{r} \times 1$ space-time block vectors of the output block stream and noise, respectively, and $\mathbb{H}$ is an $M N_{t} \times M N_{r}$ block-diagonal channel matrix. Because of the block-circulant property of the space-time channel matrix $\mathbb{H}$, a Rician frequency-selective MIMO channel can be represented as a set of $M$ parallel independent MIMO channels [9]. One can alernatively think of an OFDM system with $M$ sub-carriers, where each sub-band is affected by flatfading. Therefore, the ergodic capacity of an equivalent OFDM 
system is equal to the sum of the ergodic capacities for each sub-band, i.e.,

$$
C_{N_{t}, N_{r}}=\frac{1}{M} \sum_{k=0}^{M-1} C_{k}
$$

where

$$
C_{k}=\max _{t r \mathbf{R}_{x x}[k] \leq P} E_{\mathbf{H}_{E}[k]}\left\{\log \operatorname{det}\left(\mathbf{I}_{N_{r}}+\mathbf{H}_{E}[k] \mathbf{R}_{x x}[k] \mathbf{H}_{E}^{H}[k]\right)\right\},
$$

and $\mathbf{H}_{E}[k]$ denotes the channel matrix corresponding to the $k$ th subband. Depending on the amount of CSI at the transmitter, $\mathbf{R}_{x x}[k]$ can be determined as optimal (capacity-achieving) or sub-optimal (resulting in information rates below the capacity) as discussed earlier.

Figures 8 and 9 illustrate the ergodic capacity for two cases: an $N_{t} \times 1$ MISO system and a $2 \times 2$ MIMO system. In each case, the experimental channel with four significant taps (see Fig.4) is used to obtain the rank-1 matrix of the mean values in the expression (14). Since for each significant tap, Rician $k$-factor is on the order of 10 , we present flat-fading results for $k=10$ as reference. We assume negligible correlation between different transmitter-receiver pairs $\left(\boldsymbol{\Theta}_{R, l}=\mathbf{I}_{N_{r}}\right.$ and $\left.\boldsymbol{\Theta}_{T, l}=\mathbf{I}_{N_{t}}\right)$. Therefore, numerical integration results should be regarded as idealized, since a realistic UWA system could have non-negligible spatial correlation at both the transmitter and receiver [14], which will degrade the system capacity.

Figure 8 illustrates the ergodic capacity for an $N_{t} \times 1$ system using the sub-optimal signaling scheme given by the expression (18). The information rates obtained by suboptimal signaling scheme are close to the capacity bound [12], especially for values of $k$ on the order of 10 or more. Results for Rayleigh fading are provided as a reference, and we can observe that the ergodic capacity is clearly greater in the Rician case. Nakagami and log-normal fading models are not considered for the sub-optimal scheme.

Figure 9 illustrates achievable information rates for different fading distributions under normalized second and fourth moments of the underlying distributions. Note that the Rician frequency-selective channel given in (20) shows poorer performance than idealized flat-fading scenarios as well as Rayleigh frequency-selective channel. Performance will be degraded since the presence of the specular components in the rank-1 matrix of the mean values is not exploited optimally. This is especially emphasized for large Rician $k$-factors when the channel matrix in (13) becomes ill-conditioned. Therefore, even though a Rician channel can potentially provide greater capacities, we are not able to exploit this advantage due to the lack of the channel knowledge at the transmitter side. At the same time, Nakagami and log-normal fading will provide wellconditioned channel matrices, resulting in similar capacities due to matching of the higher moments. We should also note that frequency-selectivity of the channel will not degrade system performance in the case of Rayleigh fading. This is true even for the Nakagami and log-normal channel because of the well-conditioned channel matrices, and matching of the higher moments will lead to the performance close to

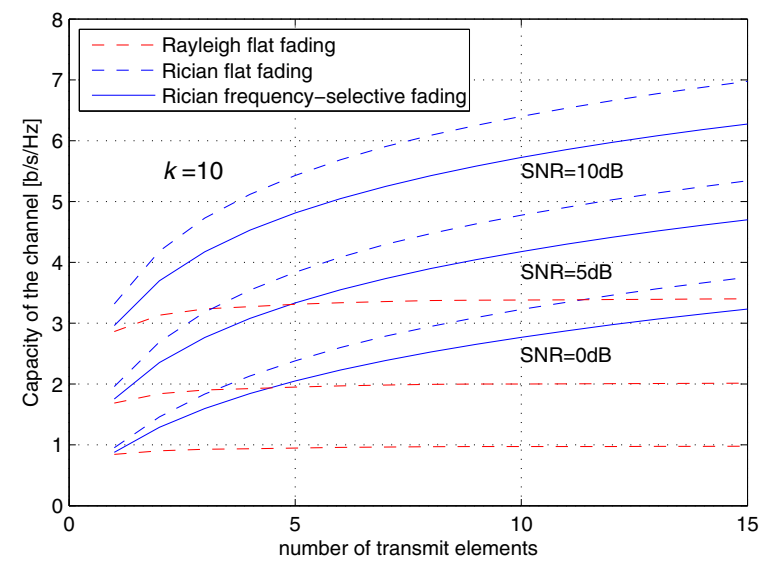

Fig. 8. Ergodic capacity of an $N_{t} \times 1$ system for the sub-optimal signaling scheme defined by the expression (18).

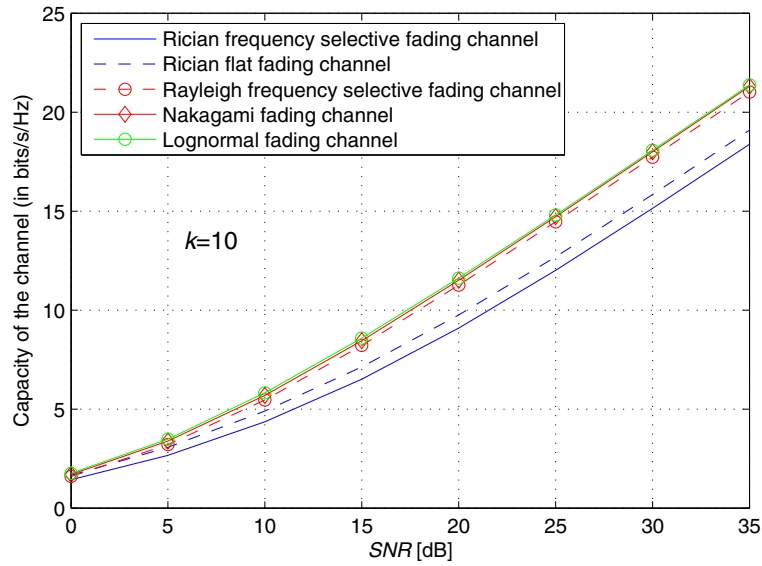

Fig. 9. Ergodic capacity of a $2 \times 2$ system with no CSI at the transmitter.

the Rayleigh fading scenario (both flat-fading and frequencyselective). Therefore, assuming the Rician model for an UWA channel, a system designer can exploit advantageous statistical knowledge of the channel at the transmitter side by implementing a receiver-transmitter feedback, and conveying information about slowly-varying statistical parameters of the underlying Rician fading distribution (e.g., the distribution mean, the variance and/or Rician $k$-factor). By designing adaptive spacetime coding techniques which utilize these fading parameters the performance of a MIMO UWA system can be significantly improved.

\section{CONCLUSion}

In this paper we considered short-term statistical characterization of the shallow water acoustic communication channel. A wideband single-carrier channel probe signal was found to offer satisfactory channel estimates. Channel measurements were used to examine several candidate fading models, indicating a good match with the Rician distribution. These measurements were also used to compute the average 
channel capacity assuming a quasi-stationary fading and nonwhite Gaussian noise. Finally, we discussed the bounds on the MIMO channel capacity under different fading models, assuming either no channel information at the transmitter, or limited knowledge of the channel statistics. Statistical knowledge about the channel fading at the transmitter side could be a useful parameter in system design. Implementing receiver-transmitter feedback in the system, information about slowly-varying mean, variance and/or Rician $k$-factor can be conveyed. Utilizing these parameters through adaptive spacetime coding techniques, the system capacity can be improved significantly.

\section{REFERENCES}

[1] M. Stojanovic, "Underwater Acoustic Communications: Design Considerations on the Physical Layer," IEEE/IFIP Fifth Annual Conference on Wireless On demand Network Systems and Services (WONS 2008), Garmisch-Partenkirchen, Germany, January 2008.

[2] G. J. Proakis, Digital Communications, 4th ed. New York: McGraw-Hill, 2001.

[3] H. M. Kwon and T. Birdsall, "Channel capacity in bits per Joule," IEEE J.Oceanic Eng., vol.11, No.1, pp.97-99, Jan. 1986.

[4] H. Leinhos, "Capacity calculations for rapidly fading communications channels," IEEE J.Oceanic Eng., vol.21, No.2, pp.137-142, Apr. 1996.

[5] M. Chitre, "A high-frequency warm shallow water acoustic communications channel model and measurements," JASA, 122(5), pp.2580-2586, Nov 2007.
[6] M. Stojanovic, "On the Relationship Between Capacity and Distance in an Underwater Acoustic Communication Channel," ACM SIGMOBILE Mobile Computing and Communications Review (MC2R), vol.11, Issue 4, pp.34-43, October 2007.

[7] I. E. Telatar, "Capacity of multi-antenna Gaussian channels," European Transactions on Telecommunications, vol.10, pp.585-595, Nov. 1999

[8] G. J. Foschini and M. J. Gans, "On limits of wireless communications in a fading environment when using multiple antennas," Wireless Personal Communications, vol.6, pp.311-335, 1998

[9] L. Pillutla and S. K. Jayaweera, "MIMO capacity of an OFDM-based system under Ricean fading," IEEE Vehicular Technology Conference (VTC - spring), Milan, Italy, May 2004.

[10] H. Bolcskei, D. Gesbert, and A. Paulraj, "On the capacity of OFDMbased spatial multiplexing Systems," IEEE Transactions on Communications, vol.50, No.2, pp.225-234, February 2002.

[11] S. Jin, X. Gao, and X. You, "On the Ergodic Capacity of Rank-1 RiceanFading MIMO Channels," IEEE Transactions on Information Theory, vol.53, No.2, pp.502-517, February 2007.

[12] S. K. Jayaweera and V. H. Poor, "On the capacity of multiple-antenna systems in Rician fading," IEEE Transactions on Wireless Communications, vol.4, No.3, pp.1102-1111, May 2005.

[13] R. J. Turyn, "Sequences with small correlation," In Error Correcting Codes: Proceedings of a Symposium (H. B. Mann, ed.), New York: Wiley, pp.195-228, 1968.

[14] L. A. Pflug, G. E. Ioup, J. W. Ioup, P. Jackson, "Variability in higher order statistics of measured shallow-water shipping noise," Proc. of the IEEE Signal Processing Workshop on Higher-Order Statistics, pp.400404, July 1997. 